\title{
Molecular Elasticity and the Geometric Phase
}

\author{
Joseph Samuel and Supurna Sinha \\ Raman Research Institute, Bangalore 560080,India
}

(November 12, 2018)

\begin{abstract}
We present a method for solving the Worm Like Chain (WLC) model for twisting semiflexible polymers to any desired accuracy. We show that the WLC free energy is a periodic function of the applied twist with period $4 \pi$. We develop an analogy between WLC elasticity and the geometric phase of a spin $\frac{1}{2}$ system. These analogies are used to predict elastic properties of twist-storing polymers. We graphically display the elastic response of a single molecule to an applied torque. This study is relevant to mechanical properties of biopolymers like DNA.
\end{abstract}

PACS numbers: 82.37.-j,03.65.Vf,87.14.-G,87.15.-V

Some long molecules are as stiff as needles, others as flexible as thread. The elasticity of a stiff molecule is dominated by energy, while the elasticity of a flexible molecule is dominated by its configurational entropy. It has lately become feasible [1] to stretch, bend and twist single molecules to study their elastic properties. The subject of this paper is the elasticity of semiflexible polymers in which there is competition between energetic and entropic effects. We consider a polymer which can bend as well as twist [2]. The flexibility of such a molecule is characterized by two dimensionless parameters $\alpha=L_{B P} / L_{T P}$ and $\beta=L / L_{B P}$, where $L$ is the length of the molecule and $L_{B P}$ and $L_{T P}$ are the bend and twist persistence lengths. For example DNA has a bend persistence length of about $53 \mathrm{~nm}$ and a twist persistence length of about $70 \mathrm{~nm}$.

The main purpose of this letter is to draw attention to an experimentally relevant topological subtlety which has not been discussed in previous theoretical treatments of the elasticity of twisting polymers. To appreciate the point, take a strip of paper (a ribbon or belt will do as well) and tape one end of the strip to a table. Pull the strip taut by its other end and twist it by four half-turns ( $4 \pi$ rotation). If you now slacken the strip, you will find that it is possible, keeping the end fixed, to pass the strip around the end. Pulling the strip taut will reveal that the $4 \pi$ twist has been released. This demonstration shows that a polymer can release twist, two turns at a time by going around its end. A twist of $2 \pi$ cannot be so released but can be transformed to $-2 \pi$. This of course, merely illustrates the well known mathematical fact that the rotation group is doubly connected [3]. We will now see that this mathematical fact has concrete experimental consequences for the elastic properties of twist-storing polymers. Mentally replace the table with a translation stage, the strip with DNA molecule with a micron sized magnetic bead at its free end and let the twisting be done with a magnetic field. If the DNA molecule is about $16 \mu$ long, and not pulled taut, it can release $4 \pi$ worth of twist by passing around the bead. One will find that twisting the bead by $4 \pi$ is equivalent to not twisting it at all! Under the influence of thermal agitation, the molecule will explore all configurations which it can reach by continuous deformation. As we have shown, a configuration with a $4 \pi$ twist can be continuously deformed to a configuration with 0 twist. It follows that the free energies for these two situations are the same. If one were to measure the free energy $G(\psi)$ of the molecule as a function of applied twist $\psi$ (by say measuring the torque-twist relation), one would find that $G(\psi)$ is a periodic function of $\psi$ with period $4 \pi$. From the $4 \pi$ periodicity of the free energy, it follows that the torque-twist relation and other measurable elastic properties have the same periodicity.

The above discussion is even more relevant to theoretical models of polymers which do not incorporate self avoidance. Such a polymer is a 'phantom chain' [4] and can pass through itself. In the absence of self avoidance, a polymer does not even need to pass around its end: it can pass through itself and so release twist two turns at a time. Indeed, such effects have been seen in recent single molecule experiments [5] and even earlier [6]: the enzyme topoisomerase II converts a real DNA into a 'phantom chain' $[5,7]$ and in the presence of this enzyme (which plays a crucial role in replication), the DNA molecule releases twist two turns at a time $[5,6]$. The release of twist through bending modes (geometric untwisting) has also been discussed in [8].

It is thus clear that in theoretical models which do not have self avoidance, the free energy is a periodic function of applied twist with period $4 \pi$. Bearing this in mind, we will now explore the Worm Like Chain (WLC) [9] model for twisting polymers, which ignores self avoidance. As was emphasized recently [10] in a review of single molecule experiments, "the precision of control and quantitative measurement and simple interpretation of these experiments make detailed theoretical analyses appropriate". While there has been some theoretical progress $[4,11]$ on the WLC for twisting polymers, interest has been confined primarily to the high tension regime, which is theoretically more tractable because the molecule has only small perturbations about a linear configuration. Experiments of Bensimon [12] have also explored this regime. In contrast the present letter theoretically explores the non linear regime of small forces, 
where the perturbative methods described in $[4,11]$ are inapplicable. In this regime we find that the WLC free energy has a $4 \pi$ periodicity in contrast to the aperiodic free energy of the high tension regime.

We first describe the WLC model and summarize the existing theoretical analyses of this model. We then point out a physically relevant mathematical subtlety without which the solution of the WLC model is incorrect. We then correctly solve the WLC model by a combination of analytical and numerical techniques and elucidate some of the elastic properties that emerge from the model in graphical form. A concluding discussion interprets the results.

WLC Model: The WLC model ignores self avoidance and views the polymer as a framed space curve $\mathcal{C}=\left\{\vec{x}(s), \hat{e}_{i}(s)\right\}, i=1,2,3,0 \leq s \leq L$ of contour length $L$, with an energy $\operatorname{cost} \mathcal{E}(\mathcal{C})$ for bending and twisting. We suppose that one end of the polymer is tethered to the origin $\vec{x}(0)=0$ and the other end at $\vec{x}(L)=\vec{r}$ is tagged. The unit tangent vector $\hat{e}_{3}=d \vec{x} / d s$ to the curve describes the bending of the polymer while the twisting is captured by a unit vector $\hat{e}_{1}$ normal to $\hat{e}_{3}$. $\hat{e}_{2}$ is then fixed by $\hat{e}_{2}=\hat{e}_{3} \times \hat{e}_{1}$ to complete the right handed moving frame $\hat{e}_{i}(s), i=1,2,3$. The rate of change of the moving frame $\hat{e}_{i}(s)$ along the curve can be measured by its "angular velocity vector" $\vec{\Omega}$ defined by

$$
\frac{d}{d s} \hat{e}_{i}(s)=\vec{\Omega} \times \hat{e}_{i}(s) .
$$

The components of $\vec{\Omega}$ in the moving frame are $\Omega_{i}=\vec{\Omega} \cdot \hat{e}_{i}$ and the energy $\mathcal{E}(\mathcal{C})$ of a configuration $\mathcal{C}$ is given by

$$
\mathcal{E}[\mathcal{C}]=1 / 2 \int_{0}^{L}\left[A\left(\left(\Omega_{1}\right)^{2}+\left(\Omega_{2}\right)^{2}\right)+C\left(\Omega_{3}\right)^{2}\right] d s,
$$

where $A$ is the bending modulus and $C$ the twist modulus.

Imagine that the ends of the polymer and the frames at these ends $\left(\hat{e}_{i}(0), \hat{e}_{i}(L)\right)$ are held fixed. We wish to compute the number of configurations

$$
Q\left(\vec{r}, \hat{e}_{i}(0), \hat{e}_{i}(L)\right)=\Sigma_{\mathcal{C}} \exp (-\mathcal{E}[\mathcal{C}] / k T),
$$

counted with Boltzmann weight $\exp (-\mathcal{E}[\mathcal{C}] / k T)$, which start at the origin with initial frame $\hat{e}_{i}(0)$ and end at $\vec{r}$ with final frame $\hat{e}_{i}(L)$. The function $Q$ is related to the free energy of the molecule and its measurable elastic properties like the force-extension relation (FER) and the torque-twist relation (TTR). An overall multiplicative constant is not important in the calculation of $Q$. This only leads to an additive constant in the free energy, which drops out on differentiation and does not affect elastic properties.

Let us fix a "lab frame" $\tilde{e}_{i}^{b}$ and write $e_{i}^{a}(s)=R_{b}^{a}(s) \tilde{e}_{i}^{b}$, where $a=1,2,3$ is a vector index (as opposed to the frame index $i$ ) and $R(s) \in S O(3)$ is a $3 \times 3$ rotation matrix. There is a clear analogy between the elastic properties of the WLC and the motion of a top. Indeed as Mezard and Bouchiat [11] and Moroz and Nelson [4] point out, the WLC problem can be mapped to the quantum mechanics of a symmetric top. From this mapping, one may naively conclude [13] that the periodicity of the free energy is $2 \pi$. We now show that a careful treatment of the path integral (3) gives the correct $4 \pi$ periodicity.

Each configuration $\mathcal{C}$ in (3) is characterized by a curve $\{R(s)\}$ in the rotation group with fixed end points $R(0)$ and $R(L)$. The sum is over all configurations which are sampled under the influence of thermal agitation. It is evident that we should only sum over configurations $\{R(s)\}$ in a single homotopy class: thermal agitation only causes continuous deformations and therefore cannot knock the polymer out of its homotopy class. $Q$ thus depends not only on $(\vec{r}, R(0), R(L))$, but also on the homotopy class $[\{R(s)\}]$ of the paths $\{R(s)\}$ being summed over. This information is exactly captured by going to the covering space $S U(2)$ of the rotation group $S O(3)$. This step is essential to correctly describe the elastic properties of the twisting polymer. (If we do not take this step but remain on $S O(3)$, we are effectively summing over both homotopy classes, which is a physically incorrect procedure.) The result is that while the WLC Hamiltonian is the same as that of the top, the WLC configuration space is not the configuration space $S O(3)$ of the top, but its double cover $S U(2)$ [3]. As we will see below, this results in a $4 \pi$ periodicity for the free energy. $S U(2)$ is the same as $S^{3}$, the four dimensional sphere defined by $\left\{x^{\alpha}, \alpha=1,2,3,4,\right\}, \Sigma_{\alpha}\left(x^{\alpha}\right)^{2}=1$. In fact, $x^{\alpha}$ are the Cayley-Klein parameters [14] traditionally used in describing tops.

Let $\{g(s)\}$ be a continuous curve in $S U(2)$ which maps down to the curve $\{R(s)\} . g(s)$ satisfies the differential equation $\frac{d g(s)}{d s}=i / 2(\vec{\Omega} . \vec{\sigma}) g(s)$, whose solution is a path ordered exponential: $g(s)=P\left[\exp \int_{0}^{s} i \vec{\Omega}\left(s^{\prime}\right) \cdot \vec{\sigma} / 2 d s^{\prime}\right] g(0)$. $\mathcal{C}$ is now described by a curve $\{g(s\}$ in $S U(2)$, with fixed end points $g(0)$ and $g(L)$. The standard Euler angles $(\theta, \phi, \psi)$ on the rotation group can be used as co-ordinates on $S U(2)=S^{3}$ if the range of $\psi$ is extended to $4 \pi$ [3]. $S U(2)$ acts on itself by right and left action generated [15] by space fixed $\left(J_{x}, J_{y}, J_{z}\right)$ and body fixed $\left(J_{1}, J_{2}, J_{3}\right)$ angular momenta.

We can now write $(3)$ more correctly as $Q\left(\vec{r}, q_{0}, q_{L}\right)$ where $q_{0}=g(0)$ and $q_{L}=g(L)$, to explicitly display the homotopy class dependence of $Q . Q\left(\vec{r}, q_{0}, q_{L}\right)$ has the path integral representation:

$$
\mathcal{N} \int D[g(s)] e^{\left[-\mathcal{E}(\mathcal{C}) / k_{B} T\right]} \delta(\vec{x}(L)-\vec{r}) .
$$

$\mathcal{N}$ is a normalisation constant and the path integral is over all paths that go from $q_{0}$ to $q_{L}$ on $S^{3}$. We now pass from $Q\left(\vec{r}, q_{0}, q_{L}\right)$ to its Laplace transform defined 
as $\tilde{Q}\left(f, q_{0}, q_{L}\right)=\int d \vec{r} \exp \left[\vec{f} \cdot \vec{r} / L_{B P}\right] Q\left(\vec{r}, q_{0}, q_{L}\right)$, where $L_{B P}=A / k T$. Performing the elementary integrations and changing variables to $\tau=s / L_{B P}$ and $\vec{\omega}=\vec{\Omega} L_{B P}$, we see that $\tilde{Q}\left(f, q_{0}, q_{L}\right)$ can be represented as $\mathcal{N} Z\left(f, q_{0}, q_{L}\right)$, where $Z$ has the path integral representation

$$
\int D[g(\tau)] e^{-\left[\int_{0}^{\beta} d \tau \frac{1}{2}\left(\omega_{1}^{2}+\omega_{2}^{2}+\alpha^{-1} \omega_{3}^{2}\right)-\vec{f} \cdot \hat{e}_{3}\right]},
$$

where $\alpha=A / C=L_{B P} / L_{T P}$. This is clearly the quantum amplitude $<q_{L}\left|\exp \left[-\beta H_{f}\right]\right| q_{0}>$ for a particle on the surface of a 3 -sphere to go from an initial position $q_{0}$ on $S^{3}$ to a final position $q_{L}$ in imaginary time $\beta$ in the presence of an external force field. The Hamiltonian is $H_{f}=H_{0}-f \cos \theta$, where $H_{0}=1 / 2\left({J_{1}}^{2}+{J_{2}}^{2}+\alpha{J_{3}}^{2}\right)$, which is the Hamiltonian of a symmetric top. If the exact eigenstates of $H_{f}$ were known, we could write:

$$
Z=\Sigma_{n} \exp \left[-\beta E_{n}\right] u_{n}^{*}\left(q_{0}\right) u_{n}\left(q_{L}\right),
$$

where $\left\{u_{n}(q)\right\}$ is a complete set of normalised eigenstates of the Hamiltonian $H_{f}$ and $E_{n}$ are the corresponding eigenvalues. Even though $H_{f}$ cannot be diagonalised analytically, we can exploit its symmetries to reduce the problem to a numerically tractable form. $J_{z}$ and $J_{3}$ commute with the Hamiltonian $H_{f}$, reflecting the symmetry under space-fixed and body fixed rotations about the third axis. As a result, $Z$ depends only on the differences $\phi=\phi_{L}-\phi_{0}$ and $\psi=\psi_{L}-\psi_{0}$ and we write $Z\left(f, \theta_{0}, \theta_{L}, \phi, \psi\right)$. Consider the dependence of $Z$ on $\psi$. Since all the wave functions in (6) are single valued functions on $S^{3}$, it follows that $Z(\psi)$ is periodic in $\psi$ with period $4 \pi: Z(\psi+4 \pi)=Z(\psi)$. This means that the free energy $G=-1 / \beta \log [Z]$ and all elastic properties have the same period. This is the first main result of this letter. The fact that the periodicity is $4 \pi$ and not $2 \pi$ can be traced to the fact the sum in (6) extends not only over tensorial states but also over spinorial ones. The variation of $G$ with respect to the variables $\left(f, \theta_{0}, \theta_{L}, \phi, \psi\right)$ gives the elastic response to stretch $(f)$, bend $(\theta, \phi)$ and twist $(\psi)$. $G$ can be computed numerically for any value of its arguments using mathematica programs [16] that run for a few minutes on a PC, using methods similar to those of [17]. From $G$ we can extract all possible information regarding the elasticity of a polymer with bend and twist degrees of freedom. For instance one can predict the form of extension versus twist curves for various values of the stretching force.

The $4 \pi$ periodicity of the free energy strongly motivates the use of spinorial methods. In fact, the WLC configuration space $S^{3}$ is the same as the set of normalised states of a spin-1/2 quantum system. We will now show that there is a mapping between a configuration of a twisting polymer and the quantum evolution of a spin- $1 / 2$ system. This brings out an interesting connection between $W L C$ elasticity and the Geometric Phase. Let us introduce a 2 component complex vector (a spinor) $\xi^{1}=x^{1}+i x^{2}, \xi^{2}=x^{3}+i x^{4}$, which is normalised $\left(\xi^{\dagger} \xi=1\right)$. We can write $\xi^{1}=$ $\cos \theta / 2 \exp ^{-i \phi / 2} \exp ^{i \psi / 2}, \xi^{2}=\sin \theta / 2 \exp ^{i \phi / 2} \exp ^{i \psi / 2}$ and thus introduce co-ordinates $(\theta, \phi, \psi)$ ranging from 0 to $(\pi, 2 \pi, 4 \pi)$ respectively. These are similar to Euler angles on the rotation group and differ only in the range of $\psi$. The frame $\hat{e}_{i}$ can be expressed as $\hat{e}_{3}=\xi^{\dagger} \vec{\sigma} \xi$, $\hat{e}_{1}+i \hat{e}_{2}=\xi^{T}\left(i \sigma_{2}\right) \vec{\sigma} \xi$, where the $\sigma \mathrm{s}$ are the usual Pauli matrices. Notice that altering $\psi$ by $2 \pi$ flips only the sign of $\xi$ and therefore does not affect the frame. Using this mapping between 2 component spinors and frames, we can import ideas from the geometric phase to understand WLC elasticity. The information in the spinor $\xi$ can be decomposed into an overall phase $\psi / 2$ describing twist and a ray $\hat{e}_{3}$ describing bend. Fix a configuration $\mathcal{C}$ and note that $\xi(s)=g(s) \xi(0)$ satisfies the Schrödinger differential equation $i \frac{d \xi(s)}{d s}=\hat{h} \xi(s)$, where $\hat{h}=-\vec{\Omega} \cdot \vec{\sigma} / 2$ is the "Hamiltonian" of a spin half particle in an external magnetic field $\vec{\Omega}$. We can now decompose the difference $\psi / 2=\psi(L) / 2-\psi(0) / 2$ between the final and initial phases into a geometric phase and a dynamical phase. The dynamical phase is given by the integral of the expectation value of the "Hamiltonian" $\int_{0}^{L} d s \xi^{\dagger} \hat{h} \xi$, which, using the definition of $\hat{e}_{3}$ above is seen to be $\int_{0}^{L} d s \Omega_{3} / 2$, half the twist $\Omega_{3}$ integrated along the polymer. The geometric phase is given by half the solid angle swept out by the ray $\hat{e}_{3}(s)$. If the initial and final rays are distinct (but not antipodal), one can join them by the unique shorter geodesic [18] to enclose a solid angle. The total twist difference $\psi$ is the sum of the "dynamical twist"-the integrated local twist- $\int \Omega_{3} d s$ and the "geometric twist"the solid angle swept out by the tangent vector- which depends on the bending of the polymer. In the literature $[4,11,19]$, the distribution of applied twist between twisting and bending is compared with the decomposition of link into twist and writhe. This result is referred to as White's theorem [20] (though an earlier reference is [21]). The discussion $[4,11,19]$ applies to closed, self-avoiding polymers which have been twisted an integral number of times. In contrast, our treatment applies also to open polymers which have been twisted a fractional number of times. However, since the WLC model does not take into account self avoidance, a twist of $4 \pi$ is equivalent to no twist and the integral part of the twist is only measured modulo two. Our treatment captures the fractional part of the applied twist (which is geometrical) and the earlier treatment captures its integral part (which is topological). In this sense, the two discussions are complementary. The analogy between twist elasticity and the geometric phase is the second main result of this letter. The analogy has also been noted in Ref [19], which, however, uses a vectorial correspondence rather than a spinorial one. The decomposition of applied twist into a geometrical and a dynamical part leads to a coupling between the bend and the twist degrees of freedom and has a direct bearing on the elastic properties of the WLC 
polymer. As a specific illustration we give the results for the special case of pure twist elasticity.

Pure twist elasticity: We suppose that the tagged end is not constrained in position, but only in orientation. Integrating $Q\left(\vec{r}, q_{0}, q_{L}\right)$ over $\vec{r}$, we see that the applied force $f$ vanishes. We also suppose that the initial $\hat{e}_{3}(0)$ and final $\hat{e}_{3}(L)$ tangent vectors are both in the same direction (which we take to be the $z$ direction). We compute the distribution $Z(\psi)$ of $\psi$. In this case only states for which $m=g$ contribute [22] and $Z$ takes the form $Z=\Sigma_{g} \mathcal{Z}_{g g}$. Using standard techniques from angular momentum theory, we can express $Z$ as $Z=\Sigma_{g} e^{i g \psi} Q_{g}$ where $Q_{g}=\Sigma_{j=|g|}^{\infty}(2 j+1) \exp \left[-\beta / 2\left(j(j+1)+(\alpha-1) g^{2}\right)\right]$, where $j$ runs in integer steps. By an inverse Fourier transform, we compute $P(\psi)$ and the free energy $G(\psi)=$ $-1 / \beta \log [P(\psi)]$. By differentiating with respect to $\psi$, we compute the torque $\Theta=\partial F / \partial \psi$ needed to twist the molecule by an angle $\psi$. The torque-twist relation is plotted in Fig.1. This graph, which is the third main result of this letter, describes the pure twist elastic properties of a molecule in the WLC model. These graphs are easily interpreted in terms of the geometric phase ideas described earlier. For large $\alpha$ ( $\alpha$ is the ratio of the bend to the twist persistence length), twist costs very little energy, the molecule twists without bending, and as it takes hardly any torque to twist the molecule, the TTR is almost flat. As $\alpha$ decreases, the applied twist is shared between the twist and the bend. When $\alpha$ is zero twisting is prohibitively expensive and the applied twist is all taken up by the bend. This causes the molecule to buckle just as a towel does when it is wrung. When $\alpha=0$, the "polarisation vector" $\hat{e}_{1}$ is parallel transported along the polymer. The distribution $Z(\psi)$ then reduces to the distribution of solid angles (Berry Phases) enclosed by closed Brownian paths on the (Poincare) sphere, which was calculated in [23] in the context of depolarised light scattering.

In this letter we have solved the WLC model with bend and twist degrees of freedom and noticed analogies to spin $1 / 2$ systems and the geometric phase. These analogies lead to a description in terms of a particle on a sphere in external gravitational and magnetic fields. Such analogies, apart from giving us analytic tools to solve the problem virtually exactly for the first time, also provide simple physical pictures: Imposing a twist on a molecule is like applying a magnetic field. The helical shape of a towel when it is wrung is similar to the helical trajectory of a particle in a magnetic field. We hope this letter will encourage experimental work on twisting polymers in the nonlinear low tension regime and set up a dialog between the theory and experiments on molecular elasticity.

Acknowledgements: It is a pleasure to thank A. Dhar, Y. Hatwalne B. R. Iyer, V.A. Raghunathan and M. Rao for their comments and R. Goldstein for his constructive suggestions which improved the paper and for drawing attention to related work [8].
[1] J.M. Schurr and S.B. Smith, Biopolymers 29, 1161 (1990); S.B.Smith and A.J. Bendich, Biopolymers 29, 1167 (1990); S.B.Smith et al, Science, 258, 1122 (1992). J. Marko and E. D. Siggia, Macromolecules 28, 8759 (1995). C. Bouchiat et al, Biophysical Journal 76, 409 (1999). C. Bustamante et al, Current Opinion in Structural Biology 10, 279 (2000).

[2] S. Panyukov and Y. Rabin, Europhys. Lett. 57, 512 (2002); Y. Kats, D. A. Kessler and Y. Rabin, Phys. Rev. E 65, 020801 (2002); S. Panyukov and Y. Rabin, Phys. Rev. E 62, 7135 (2000).

[3] J. J. Sakurai Modern Quantum Mechanics The Benjamin/Cummings Publishing Company, Inc. Menlo Park, California, pp 163-171; R. W. Wald General Relativity The University of Chicago Press, p 346.

[4] J.D. Moroz and P. Nelson, Macromolecules 31, 6333 (1998).

[5] T.R. Strick et al, Nature 404901 (2000).

[6] P.O.Brown and N.R. Cozarelli, Science 2061081 (1979);

[7] B. Alberts et al. Molecular Biology of the Cell Third Edition Garland Publishing, Taylor and Francis Group, pp 260-262.

[8] R. E. Goldstein et al, Phys. Rev. Lett. 80, 5232 (1998); N. H. Mendelson et al, J. Bacteriol. 177, 7060 (1995).

[9] O. Kratky and G. Porod, Recl. Tral. Chim. 68, 1106 (1949).

[10] S. Cocco et al, cond-mat/0206238.

[11] C. Bouchiat and M. Mezard, Phys. Rev. Lett. 80, 1556 (1998). C. Bouchiat and M. Mezard, Eur. Phys. J. E2, 377 (2000).

[12] T.R. Strick et al, Science 2711835 (1996).

[13] (see eq. 8 of [4]) This remark does not however, invalidate the main conclusions of Refs. [4,11]. These works are primarily interested in the large force limit of the WLC. Release of $4 \pi$ twist is then exponentially suppressed by energetics. The present study does not address this regime.

[14] H. Goldstein, Classical Mechanics, Addison-Wesley (1980), USA.

[15] L.D. Landau and E.M. Lifshitz Quantum Mechanics, Pergammon Press, Oxford (1977).

[16] Stephen Wolfram, The Mathematica Book, Third Edition (Wolfram Media/ Cambridge University Press, 1996).

[17] J. Samuel and S. Sinha Phys. Rev. E 66, 050801(R) (2002).

[18] J. Samuel and R. Bhandari Phys. Rev. Lett. 60, 2339 (1988).

[19] A.C. Maggs and V. Rosetto, Phys. Rev. Lett. 87, 253901 (2001); eprint cond-mat/0111011.

[20] J.H. White, Amer. J. Math. 91, 693 (1969); F.B. Fuller, Proc. Nat. Acad. Sci. USA bf 68, 815 (1971).

[21] G. Calugareanu, Rev. Math. Pures. Appl. 4, 5 (1959).

[22] A.R. Edmonds Angular Momentum in Quantum Mechanics Princeton University Press, Princeton (1960).

[23] S. Sinha and J. Samuel, Phys. Rev. B 50, 13871 (1994); M.M.G. Krishna et al, J. Phys. A. 335965 (2000). 
FIG. 1. The Torque-Twist relation for $\beta=L / L_{B P}=1$, $\alpha=L / L_{T P}=0,1,7$. 